\title{
Im Kampf gegen die Trägheit angesichts des Klimawandels
}

\section{Jean Martin}

Dr. med., Redaktionsmitglied

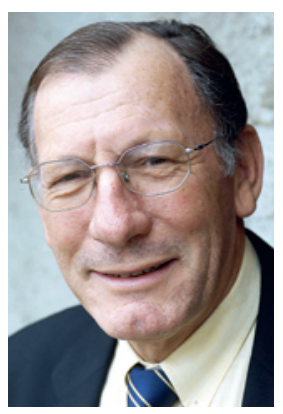

«Die in der Schweiz und andernorts in Europa auftretenden Mobilisierungen überraschen den Beobachter in Umfang, Jugend und in ihrem radikalen Ton» [1]. Die Klima-Demos / manifs pour le climat / marce per il clima vom 2. Februar haben in den Schweizer Städten ca. 40000 Teilnehmende versammelt. Auf den Hunderten von Plakaten und Transparenten war u.a. wie folgt zu lesen: "Auch die Dinosaurier dachten, sie hätten noch Zeit», "Willst du den Planeten bleu oder well done?», «Es gibt keinen Planeten B». Und politischer: «Rettet das Packeis [Wortspiel: frz. la banquise] und nicht die Banken [frz. les banques]» (es ist allseits bekannt, dass das Eis der beiden Pole mit grosser Geschwindigkeit schmilzt). Zahlreiche Aussprüche beklagen die schlafenden Politiker, auch wenn der Grosse Rat von Basel-Stadt den Klimanotstand ausgerufen hat.

Das Engagement der jungen Menschen konkretisierte sich jüngst in den von Studentengruppen vom 4 . bis zum 9. März an über zwanzig Hochschulen in der Schweiz organisierten Nachhaltigkeitswochen (Sustainability Week Switzerland, SWS - www.sustainabilityweek.ch). Die Vielzahl und Vielfalt dessen, was da auf die Beine gestellt wurde, beeindruckt. SWS-Website: «Wir glauben, dass die Hochschulen nachhaltige (sustainable) Campus anstreben, dies aber nicht realisieren können. [...] Wir wollen Nachhaltigkeit auf allen Ebenen des Hochschullebens integrieren und die Einrichtungen dabei unterstützen, eine 'role models'-Funktion für die Gesellschaft im Allgemeinen zu übernehmen. Die potentiellen Auswirkungen auf globaler Ebene sind enorm.»

Die Universität Lausanne und die EPFL thematisierten die Forderung «Das System ändern, nicht das Klima» und starteten damit am 28. Februar in die Aktionswoche. Jacques Dubochet ist überzeugt, dass die Dynamik die Seiten gewechselt hat, vor allem dank der jungen Generation. Auch wenn die Jungen gerne auf der Seite der Älteren sind, «wollen sie die Dinge in die Hand nehmen, und wir müssen in den kommenden 20 Jahren - spätestens bis 2050 - zwingend aus fossilen Energieträgern aussteigen", meint der Nobelpreisträger. Greta Thunberg sagte in Davos, dass das einzig Vernünftige das Ziehen der Notbremse sei. Dominique Bourg, einer der Debattenführer der Frankophonie, beklagt die gegenwärtig vorherrschende klimaspezifische Fahrlässigkeit und verwies auf mehrere Indikatoren, die auf eine "grosse Beschleunigung» im vergangenen Jahrhundert hinweisen [2]. Seine Antwort auf die heftige Kritik der «Klimaskeptiker-Realos» an den erforderlichen drastischen Massnahmen, die zu erheblichen Veränderungen in unserer Lebensführung führen werden (siehe [3]): «Nein, hier geht es nicht um Ökofaschismus, sondern um Mittel und Wege, die Erde bewohnbar zu halten"; ein Überschreiten der Erderwärmung um mehr als $2^{\circ} \mathrm{C}$ ist unbedingt zu vermeiden, da es sonst zu irreversiblen Entwicklungen kommen wird. So auch der jüngste Appell von 260 Forschern zur Teilnahme am weltweiten Klimastreik vom 15. März [4]. Beispiele: Die Hochschulen sind aktiv daran beteiligt, ihre Mitarbeitenden zu einer Begrenzung von Flugreisen anzuhalten (wer hätte noch vor ein paar Jahren daran zu denken gewagt?). Der Kanton Genf hat beschlossen, dass dieses Verkehrsmittel nicht mehr für Schulreisen genutzt werden kann. Auch Simonetta Sommaruga bringt starke Argumente ..., aber natürlich müssen wir die Reaktionen jener abwarten, die immer noch von einem Wachstum ohne Grenzen träumen. Kaum gesprochen wird in diesem Kontext von einem Aspekt, den ich dennoch für wichtig halte: Wir haben Angst, so ein Pastor, der im Journal der waadtländischen Liberalen eine ethische Kolumne schreibt (ohne seine Überzeugung, dass die Politiker nicht auf der Höhe des Geschehens sind, zu verbergen). Jean-François Ramelet: «Es ist unabdingbar, dass sich eine kollektive Intelligenz kundtut. Dazu müssen wir aber erkennen, dass wir alle mehr oder weniger angstbesetzt und mit einem Gefühl von Ohnmacht ob der schieren Grösse der Probleme, die uns die Wissenschaft einstimmig beschreibt, durchs Leben gehen. Aus den gegen Klimaschutz-Veranstaltungen gerichteten Sarkasmen und Diskreditierungen spricht Angst. Diese Angst muss zum Motor der Mobilisierung werden" [5]. Allerdings wissen wir, dass Glaubensmuster auch in einem von Wissenschaft und Fakten geprägten Kontext breiten Raum einnehmen - u.a. bei jenen, die nicht sehen oder hören wollen, oder anderen, die wie bestimmte Gruppen in den USA argumentieren: «Wenn Gott bei uns Kohlevorkommen geschaffen hat, sollen wir auch davon profitieren» ... Handeln wir tatsächlich vorwiegend aus Angst vor dem Unbekannten? Nicht auszuschliessen. 